\title{
A wireless telecommunications network for real-time monitoring of greenhouse microclimate
}

\author{
Giuliano Vox, ${ }^{1}$ Pierfrancesco Losito, ${ }^{2}$ Fabio Valente, ${ }^{2}$ Rinaldo Consoletti, ${ }^{2}$ \\ Giacomo Scarascia-Mugnozza, ${ }^{1}$ Evelia Schettini, ${ }^{1}$ Cristoforo Marzocca, ${ }^{3}$ Francesco Corsi ${ }^{3}$ \\ ${ }^{1}$ Department of Agricultural and Environmental Science, University of Bari; ${ }^{2}$ Microlaben S.r.lo, \\ Bari; ${ }^{3}$ Department of Electrical and Information Engineering, Polytechnic of Bari, Italy
}

\begin{abstract}
An innovative wireless monitoring system for measuring greenhouse climatic parameters was developed to overcome the problems related to wires cabling such as presence of a dense net of wires hampering the cultivation practices, wires subjected to high temperature and relative humidity, rodents that can damage wires. The system exploits battery-powered environmental sensors, such as air temperature and relative humidity sensors, wind speed and direction, and solar radiation sensors, integrated in the contest of an 802.15.4-based wireless sensors network. Besides, a fruit diameter measurement sensor was integrated into the system. This approach guarantees flexibility,
\end{abstract}

Correspondence: Giuliano Vox, Department of Agricultural and Environmental Science (DISAAT), University of Bari, via Amendola 165/A, 70126 Bari, Italy.

Tel: +39.080 .5443547 - Fax: +39.080 .5442977 .

E-mail: giuliano.vox@uniba.it

Key words: wireless sensors network, ZigBee protocol, information technologies, agriculture, fruit diameter sensors.

Acknowledgments: the authors gratefully acknowledge the support of Microlaben S.r.l. in the hardware design phase, and of Ser\&Practices S.r.l. for the constant and thoughtful exchange of ideas during the software development and testing phase.

Funding: the work described in this paper was developed within the framework of the project WGS - Wireless Greenhouse System - Sistema wireless di monitoraggio e controllo per serre supported by POR PUGLIA 2007-2013 - Asse I Linea 1.1 - Azione 1.1.2, Bando Aiuti agli Investimenti in Ricerca per le PMI.

Contributions: the authors shared programming and editorial work equivalently within the areas of their expertise.

Received for publication: 6 March 2014.

Accepted for publication: 24 July 2014.

CCopyright G. Vox et al., 2014

Licensee PAGEPress, Italy

Journal of Agricultural Engineering 2014; XLV:237

doi:10.4081/jae.2014.237

This article is distributed under the terms of the Creative Commons Attribution Noncommercial License (by-nc 3.0) which permits any noncommercial use, distribution, and reproduction in any medium, provided the original author(s) and source are credited. ease of deployment and low power consumption. Data collected from the greenhouse are then sent to a remote server via a general packet radio service link. The proposed solution has been implemented in a real environment. The test of the communication system showed that $0.3 \%$ of the sent data packed were lost; the climatic parameters measured with the wireless system were compared with data collected by the wired system showing a mean value of the absolute difference equal to $0.6^{\circ} \mathrm{C}$ for the value of the greenhouse air temperature. The wireless climate monitoring system showed a good reliability, while the sensor node batteries showed a lifetime of 530 days.

\section{Introduction}

The achievement of optimal greenhouse microclimate conditions allows higher yields, better quality and the lengthening of the production season (Bot, 2001; Bartzanas et al., 2005); moreover it improves the pest and disease control, thus reducing the use of agro-chemicals (Picuno et al., 2011; Schettini and Vox, 2012). The management of the greenhouse microclimate is strongly reliant on the control of air temperature and relative humidity inside the greenhouse (Vox et al., 2010). The achievement of optimal climate conditions relies on suitable greenhouse covering materials and equipment for climate control (Novello et al., 2000; Vox et al., 2005; Sica and Picuno, 2008; Schettini et al., 2011; Vox et al., 2012).

Any existing microclimate control equipment of the greenhouse, such as a heating or a cooling system, is then operated so as to bring the internal microclimate closer to the desired crop conditions (Papadakis et al., 2000; Vox et al., 2008). The optimal microclimate control depends on the reliable measurement of the climatic parameters in several places of the greenhouse and at different heights. Measurements of parameters such as air temperature and relative humidity require sensors connected with the control system by means of wires crossing the greenhouse; wires are subjected to an aggressive environment with high relative humidity values, thermal cycling and presence of animals such as voles that can damage the wires. Besides the presence of a dense net of wires crossing the cultivation area hampers the cultivation practices.

All these features have to be accounted for when designing a complete monitoring and control system for a greenhouse and the use of wireless monitoring systems is a suitable solution (López Riquelme $e t$ al., 2009; Matese et al., 2009; Li et al., 2010; Garcia-Sanchez et al., 2011).

The monitoring system must also have peculiar features related to its flexibility and reliability. The units composing the system, indeed, must be located in different parts of the greenhouse; wireless network and battery-powered components have to be used to avoid issues related to cabling. A suitable choice of the communication infrastructure 
and strategy is required to improve the system flexibility along with the possibility of self-configuration of the system, while preserving the main constraint related to the lowest possible power consumption.

The ZigBee protocol (Baronti et al., 2007; Nadimi et al., 2008; RuizGarcia et al., 2008; ZigBee Alliance, 2013), which is based on the IEEE 802.15.4 standard (IEEE Standard Association, 2006), has been designed to be used in several application environments, such as home and industrial automation, environmental monitoring, support for healthcare devices and so on. It offers complete network architecture for wireless sensors network (WSN). Its design is focused on low data rate and low power consumption, to guarantee maximum lifetime for battery-operated devices. The use of battery-powered wireless nodes makes it possible to easily deploy the sensor units within the greenhouse.

The general packet radio service (GPRS) uses the existing global system for mobile communications (GSM) network to transmit and receive TCP/IP based data to and from GPRS mobile devices (Valente $e t$ al., 2009b). It provides almost ubiquitous access to the Internet. Hence, it could be successfully exploited to send information about the greenhouse to a remote server in real-time.

Wireless monitoring systems have been introduced in different application environments, such as home and industrial automation or environmental monitoring, while few applications for greenhouse have been developed (Zhou et al., 2007; Li et al., 2010). The definition of the working parameters, i.e. data transmission times and power consumption, is a critical point in the design of a suitable wireless system.

Aim of this paper is the development of a reliable wireless monitoring system for greenhouses able to overcome the above-mentioned issues related to cabling. The wireless monitoring system was designed, developed and tested in real field condition; the results concerning the reliability of the system and the definition of the working parameters are presented in this paper. The system is characterised by high flexibility and can include several types of sensors; the innovative aspect also concerns the integration in the wireless system of a fruit diameter measurement sensor.

\section{Materials and methods}

\section{System overview}

The system consists of several sensor nodes for environmental monitoring connected to a central processing unit by means of an IEEE 802.15.4 based WSN. The Central Unit is equipped with a global positioning system (GPS) receiver for the greenhouse univocal identification and localisation. Finally, a GPRS link is able to guarantee almost ubiquitous radio coverage, thus establishing a reliable communication channel towards a remote server.

The system is made of several devices belonging to three different types (Valente et al., 2009a), namely: one central node (CN), one or more sensor nodes $(\mathrm{SN})$ and one or more router nodes $(\mathrm{RN})$. These devices communicate with each other by means of wireless IEEE 802.15.4 links. A fourth element, named data collection module (DCM), is a software module hosted on a remote machine that communicates with the CN via the GPRS data link. DCM represents a central logic unit able to manage several greenhouses. Each greenhouse is monitored by a WSN. A simple overview of the whole system is sketched in Figure 1.

The CN also acts as ZigBee coordinator (ZC) of the local network. The SN's have been configured as ZigBee end devices (ZED), with limited capabilities, whereas RN are configured as full function devices (FFD) (ZigBee Alliance, 2013). These features require the implementation of a multi-hop wireless network. In this network, communication between two end nodes is carried out through a number of intermediate nodes whose function is to relay information from one point to another.

\section{Central node}

The CN, depicted in Figure 2, is made of several custom hardware components embedded in a single device. The main unit is a PIC24H Microcontroller by Microchip (Microchip Technology Inc., 2014), which manages the connected peripherals and stores the data on a Secure Digital mass storage device. The main unit is also able to process the data coming from the SN's and to communicate potential dangerous situations to the central server.

The CN connects to several devices by means of different interfaces, namely:

- ZigBee interface: the ZigBee interface is a hardware module based on the MRF24J40MB IEEE 802.15.4 transceiver by Microchip. It incorporates the 16-bit PIC24F microcontroller implementing the ZigBee stack. As already stated, the $\mathrm{CN}$ acts as the only ZC of the network.

- GPRS and GPS unit: the GPRS and GPS unit is a GM862-GPS GPRS/GPS integrated receiver (Telit, 2014) by Telit. The GPRS interface is used to connect to the remote machine hosting the DCM.

- Bluetooth interface: the $\mathrm{CN}$ features a bluetooth adapter that can be used to send information coming from the SN's to the user via a mobile device, such as a handheld computer.

- Ethernet interface: the CN configuration can be modified locally with a simple web interface accessible through an Ethernet connector. The web interface allows setting the main configuration parameters of the system, such as the IP address of the DCM, and the sensor parameters (i.e. threshold values).

The block diagram of the $\mathrm{CN}$ is depicted in Figure 3.

\section{Sensor node}

The SN's are simpler devices with respect to the CN's. The SN's are battery powered and small sized, and can communicate with the CN by means of the ZigBee network. Thus, they can be deployed in different places within the greenhouse, hence allowing thorough and flexible monitoring capabilities.

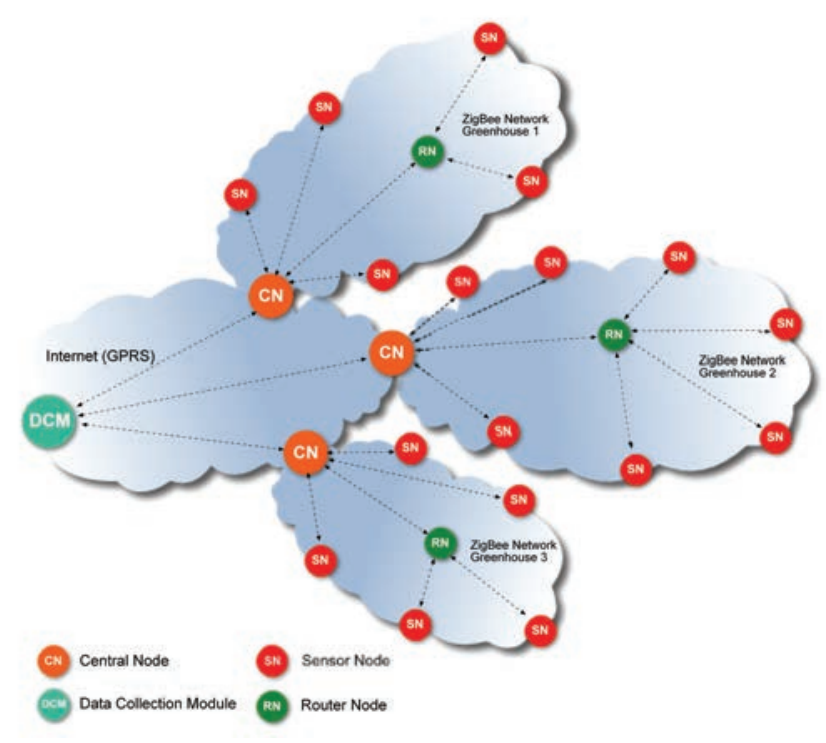

Figure 1. Architectural overview of the system. 
The SN of the WSN are designed so as to obtain the maximum flexibility in the integration of the peculiar types of sensors. In fact, the general architecture is devised to accommodate a variable number of sensors with different characteristics and includes a motherboard with end device 802.15.4 functionality and equipped with the ZigBee MRF24J40MA transceiver. Motherboard is provided with four analog input channels, a serial peripheral interface (SPI) and a universal asynchronous receiver-transmitter (UART). Sensor devices can be directly connected to the motherboard, alternatively a custom daughter board can be insert to host interface circuits.

The SN supports several types of sensors, such as:

- Temperature and humidity sensors: to monitor temperature and humidity, the Sensirion SHT75 sensor (Sensirion AG, 2014) was integrated into the systems. Both sensors are integrated onto the same circuit and coupled to 14-bit analog-to-digital converters (ADCs), with a very low power consumption ( $3 \mathrm{~mW}$ during operation, $0.005 \mathrm{~mW}$ in sleep mode); the accuracy was $0.5^{\circ} \mathrm{C}$ for temperature and $2 \%$ for relative humidity measurements.

- Air pressure sensors: the Freescale MPX4115A (Freescale Semiconductor, 2014) is an absolute air pressure sensor. This integrates on-chip, bipolar op amp circuitry and thin film resistor networks to provide a high level analog output signal and temperature compensation. Also in this case, key characteristics of such device are small size and low power consumption (35 mW during operation).

- Wind speed and direction sensor: to measure the wind intensity a Young Wind Sentry 03002 (R.M. Young Company, 1999) sensor by R. M. Young Company (Traverse City, MI, USA) was chosen. The wind velocity is measured by a classic anemometer with small rotating paddles, which produce a sinusoidal signal whose frequency is proportional to the wind speed. The wind direction is sensed by a potentiometric wind vane whose resistance is a function of the vane orientation. The measuring ranges are $0-50 \mathrm{~m} \mathrm{~s}^{-1}$ for the wind speed and $0-352^{\circ}$ for the wind direction, with an accuracy of $1.1 \mathrm{~m} \mathrm{~s}^{-1}$ and $5^{\circ}$, respectively.

- Solar radiation sensor: to measure the solar radiation intensity a pyranometer Model 8104 (Shenk GmbH, Wien, Austria) was employed whose measuring range is $0-1500 \mathrm{Wm}^{-2}$ in the wavelength range between 300 and $3000 \mathrm{~nm}$ and provides a linear output voltage of $0.015 \mathrm{mV} / \mathrm{Wm}^{-2}$ and a resolution of $1 \mathrm{Wm}^{-2}$.

- Roof aperture sensor: an analog device, ADIS16201 (Analog Devices Inc., 2014), inclinometer was selected for this purpose. This is an integrated two-axis solid-state sensor in iMEMS technology with on board signal acquisition and processing circuitry and equipped with a digital SPI output.

- Fruit diameter sensor: a fruit gauge based on a linear potentiometer was used in order to measure fruit diameter, it was interfaced to a microcontroller unit through an appropriate signal conditioning circuit. The potentiometer is fitted with a mobile metal plunger that touches the fruit with a small aluminium disc. The plunger has an electric stroke of $11 \pm 0.05 \mathrm{~mm}$ with resolution $<0.01 \mathrm{~mm}$; while microcontroller unit is provided with 10-bit ADC converter. The sensor includes a custom-built stainless steel frame (Figure 4) provided by the University of Bologna (Italy) and can be easily applied to different size fruits (Morandi et al., 2007).

\section{Router node}

The router nodes are used to forward the packets towards the CN and have also the task of widening the radio coverage of the WSN. The RN's are equipped with the PIC18F controller and the MRF24J40B transceiver.

\section{Data collection module}

The DCM is a software running on a remote machine connected to

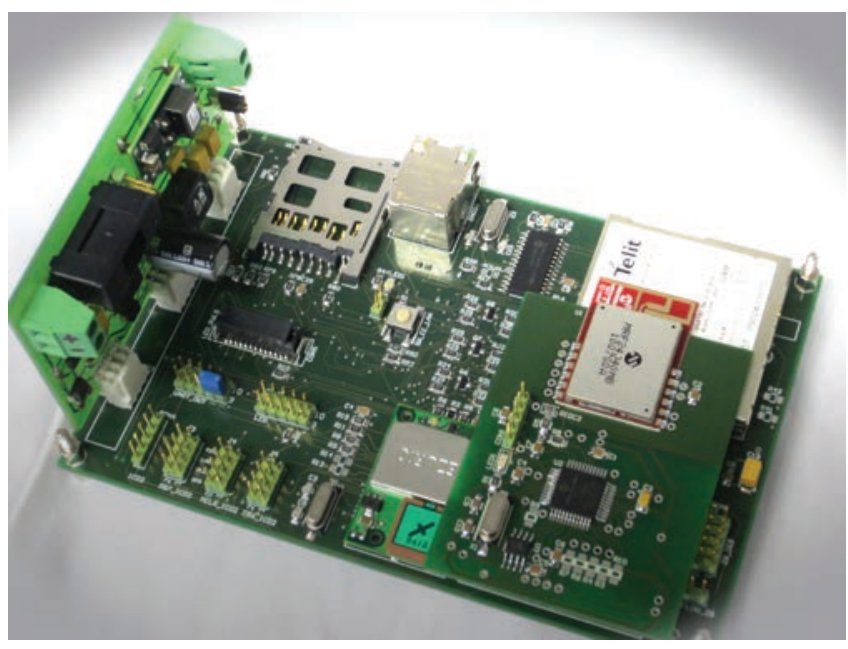

Figure 2. Central node boards.

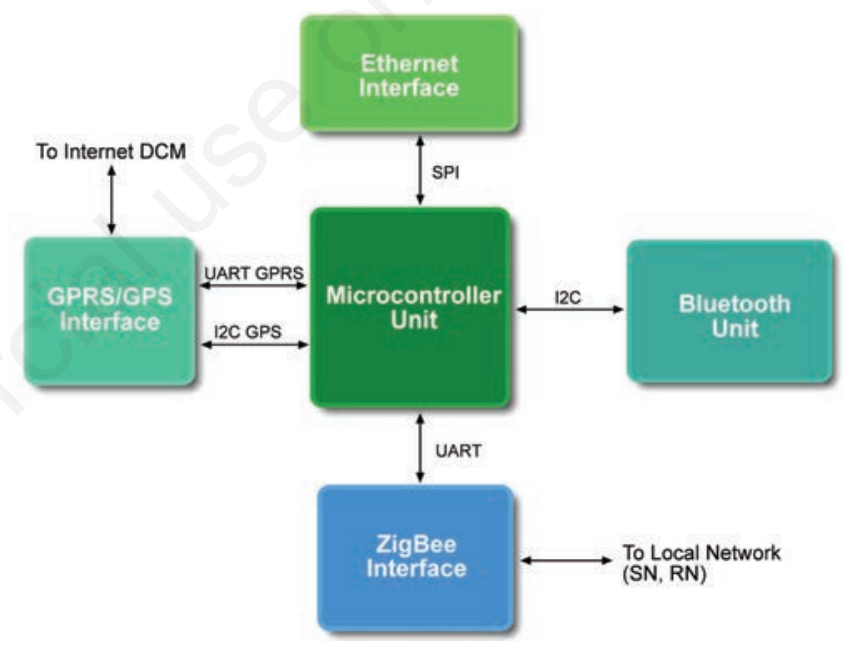

Figure 3. Central node block function diagram.

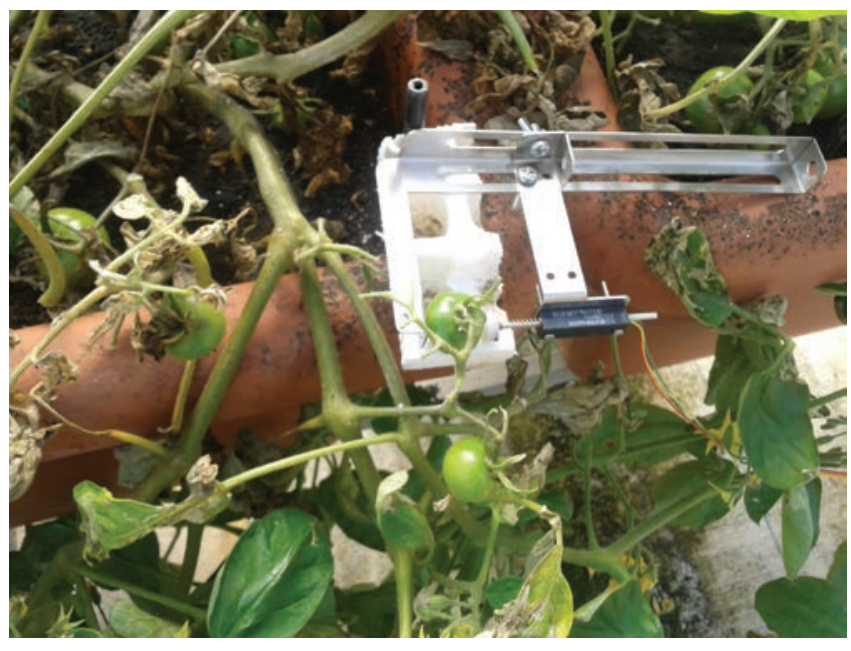

Figure 4. Fruit diameter sensor. 
the $\mathrm{CN}$ via a GPRS Internet Connection. It gathers data coming from several CN's, sends them to a central server and dispatches to the CN's the messages generated by the server. The DCM can output the processed data in several formats, and it can be run both as a standalone application or as a set of application libraries. It is made of two main components, a multithreaded server and the translation unit, and is able to manage multiple connections towards CN's at once.

When used as a standalone application, it can output data in JSON or XML formats to a local or remote data base management system (DBMS) application. On the other hand it can be used as a building block of more complex applications, providing a rich and flexible application programming interface (API), creating an abstraction layer for data formatting and interfacing to DBMS. To guarantee maximum portability, the DCM was written in Python (The Python Programming Language, 2014), a powerful general-purpose interpreted language available on many computing platforms. The DCM has been integrated into a remote web service able to monitor and control different greenhouses at once. A screenshot of the web service is depicted in Figure 5.

\section{Communication protocols and management}

\section{Communication over the ZigBee network}

The devices, which communicate over the IEEE 802.15.4 network, use small datagrams exchanged at the application layer of the ZigBee stack. Two different packet formats are used, one for the messages sent by the SN's and RN's, the other for the messages sent by the CN. The packet format is described in Figure 6. Each packet starts with a Length field ( 1 byte), which indicates the size of the payload, while the Type (1 byte) field identifies the packet type. The NodeID ( 2 bytes) is a unique identifier for each SN/RN, and the Payload field, whose size depends on the packet type, contains the actual data being sent.

At the start up, the SN/RN nodes, configured as ZED and FFD, try to associate to the $\mathrm{CN}$, i.e. the $\mathrm{ZC}$ of the network. When the wireless link is established at network level, each SN and RN announces itself to the $\mathrm{CN}$ by means of a sensor announcement packet (the type field is set to $0 x 01$ ). The payload contains information about the number and type of sensors installed in the SN and their configuration parameters. When the node is enabled, the $\mathrm{CN}$ adds the $\mathrm{SN}$ or RN to its association table and acknowledges the reception of this packet sending a message of connection-to-the-network confirmation; otherwise it refuses the connection and the node is not allowed to access the network. After this start-up phase, the CN sends an Announcement packet to the DCM, as explained later. An SN or RN is allowed to associate to an established network at any time. The Announcement packet is also sent by the CN when a new SN or RN associates or when a SN or RN de-associates from the network. Thus, the state of the network is automatically updated at runtime. After this phase, the SN starts sending data sampled from its sensor periodically, using a data sensor packet (type 0x03) directed to the $\mathrm{CN}$. The RN periodically sends to the $\mathrm{CN}$ a keep-alive packet that notifies of its presence. The $\mathrm{CN}$ can modify the rate of transmission during operation, sending a new set rate packet, either in unicast or in broadcast. The $\mathrm{CN}$ node can also send a sensor announcement request (type 0x05) if the sensor announcement has been lost or if it receives data from a $\mathrm{SN}$ or $\mathrm{RN}$, which is not included into its asso-

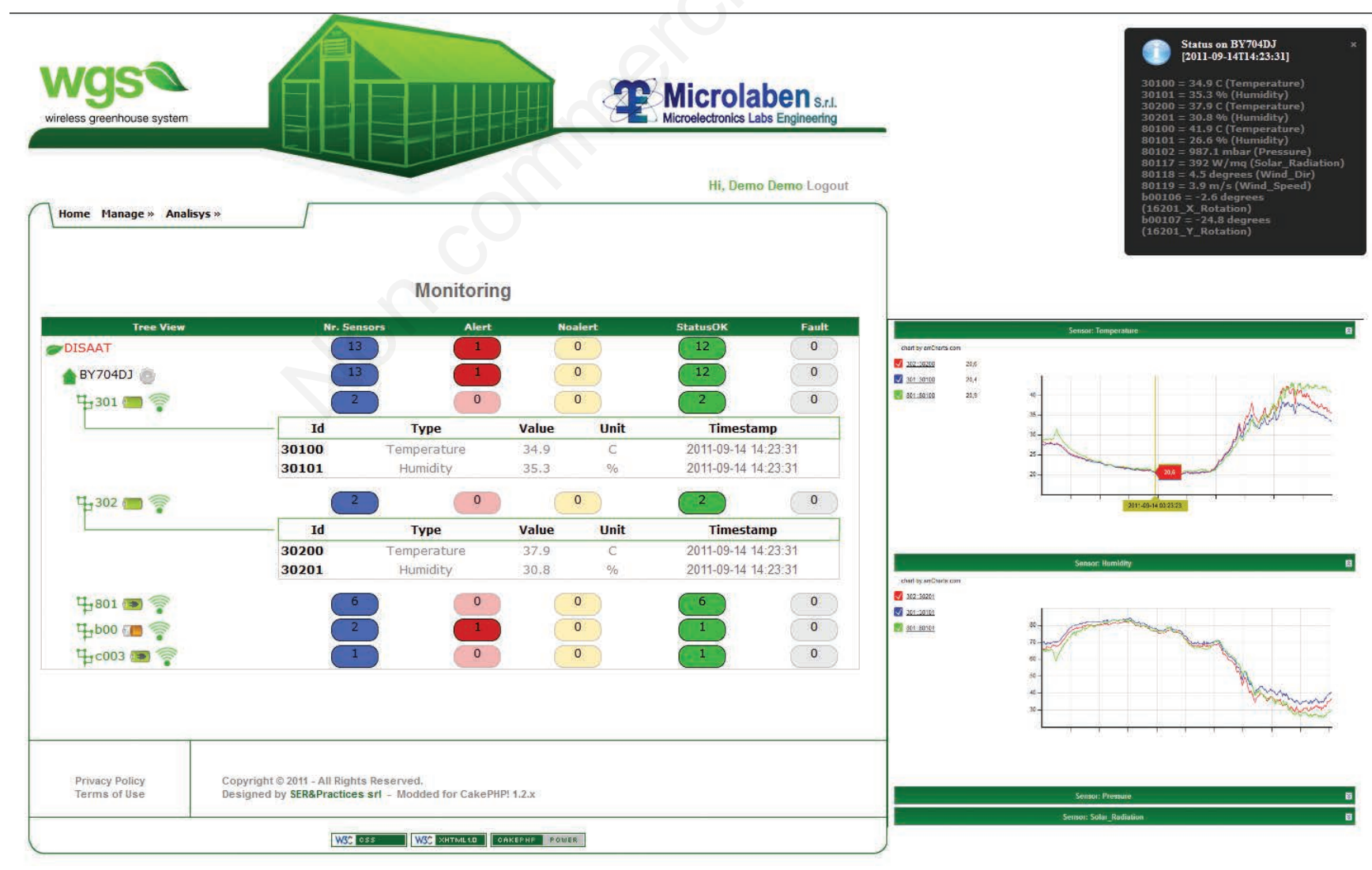

Figure 5. Web service integrating the data collection module. 
ciation table. Finally, the sensor can send a battery low packet (type $0 \times 02$ ) when the level of the batteries falls below a certain threshold.

\section{Communication over the Internet}

The communication protocol between the CN and DCM was carefully designed taking into account the characteristics and the limitations of the communication network. The GPRS (The 3rd Generation Partnership Project, 2014) offers almost ubiquitous coverage, but the data rate is rather low (theoretically up to $114 \mathrm{kbit} / \mathrm{s}$, but highly dependent on channel conditions) (Valente et al., 2009b) with highly variable transmission delays. Moreover, many mobile phone operators offer tariffs based on traffic volume. Hence, it is important to remove redundancy and compress the data in order to reduce billing costs and to increase reliability and responsiveness. We have chosen to use a simple compression mechanisms, with almost zero overhead on the $\mathrm{CN}$ and the DCM to encode and decode the information; the details are explained later in this section.

The DCM listens for incoming connections from the CN's. During the initialisation phase, the CN connects to the GPRS network and contacts the DCM. When the DCM answers, two bidirectional communication TCP sockets are opened for each connected CN, one for the data packets and one for the control messages. The former is used to deliver information from the CN to the DCM about the state of the system (sensor values), while the latter is used to interrogate and to send configuration parameters to the $\mathrm{CN}$. When the connection is established, the DCM acts as a bridge between the $\mathrm{CN}$ and a remote server or a custom user interface. The packet format is described in Figure 7. After both communication channels have been established, the $\mathrm{CN}$ sends an Announcement Packet (type 0x01) message to the DCM. This message contains detailed information about the number of SN's and RN's associated to the $\mathrm{CN}$, and also the type and number of sensors hosted on each SN. As the server knows the number of sensors hosted on each node, the $\mathrm{CN}$ only needs to send the raw data obtained by the sensors in the correct order. With this approach it is possible to reduce the amount of redundancy in the sent data, as the raw data is usually a 16bit value, smaller than the 64-bit floating point used by the DCM for internal representation. Data incoming from the nodes is transmitted at regular intervals from each $\mathrm{CN}$ to the DCM. This message is sent every $60 \mathrm{~s}$ as default value (type $0 \mathrm{x} 03$ ), though the period can be changed at runtime by the DCM. For each of the devices hosted on the associated SN's, the CN keeps a table in which the last received value and two threshold values are stored which represent the normal operation range for the sensor. If a received value falls outside the threshold values, the $\mathrm{CN}$ immediately sends to the DCM an event packet (type $0 \mathrm{x} 09$ ), with a list of all the devices that have reported an anomalous

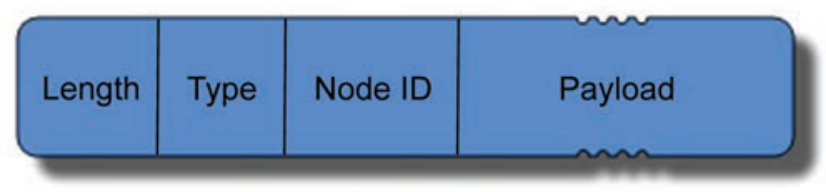

A) From Sensor Node to Central Node

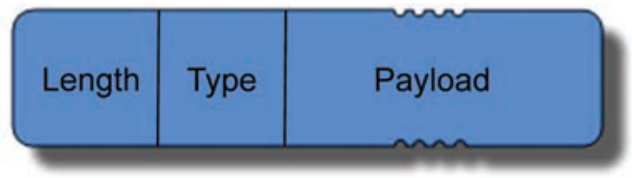

B) From Central Node to Sensor Node

Figure 6. Packet format used within the ZigBee network. value and the event type. When the value of the device, which triggered the event packet transmission, falls again into the normal operation range, the $\mathrm{CN}$ signals the event to the DCM by means of a new event packet (type 0x09). The protocol also defines several messages for communications on the control connection. All the messages are 4 bytes long strings (the identifiers are omitted herein), with the exception of the set threshold and set parameter messages, which also have a trailing payload:

- Announcement request: the announcement request message is sent when the remote server asks the $\mathrm{CN}$ for a new announcement message. This can happen when the configuration of the nodes does not match the internal database of the server, or if the latter has stale information after a node reconfiguration.

- Data request: if the remote server needs an update of the value of the sensors, it can send a status request message to the $\mathrm{CN}$, which will answer immediately (i.e., it will not wait for the periodic timer to expire).

- Node configuration request: with this message the remote server can ask the $\mathrm{CN}$ about the parameter currently set for each of the nodes such as: thresholds of the SN's, rate of network polling, rate of data packet. The $\mathrm{CN}$ responds on the data connection with a node configuration packet (type $0 \mathrm{x} 0 \mathrm{~B}$ ) containing all the parameter values for each node belonging to the network.

- General settings request: the general settings request message is sent when the server wants to know the global configuration parameters of the CN's. The CN responds on the data connection with a general settings packet (type $0 x 13$ ) containing all the configuration parameters.

- General configuration setting: the server can modify one or more global configuration parameters by means of general configuration setting message. The $\mathrm{CN}$ responds on the data connection with a general configuration packet, containing all the updated values.

- Device configuration setting: with this message the server can modify the threshold values for the sensors installed on one or more SN associated to the $\mathrm{CN}$. The $\mathrm{CN}$ responds on the data connection with a device configuration packet as in the previous case, including the updated values.

- Node configuration setting: the server can modify one or more parameters in the Nodes configuration such as network polling period, data packet period, transmission power level by means of a set node configuration setting message. The $\mathrm{CN}$ responds on the data connection with a node configuration packet, containing all the updated values.

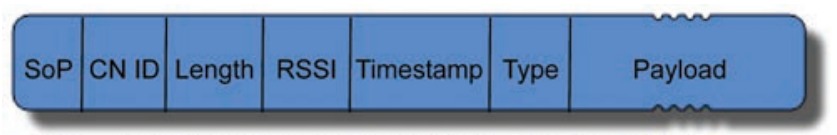

A) From Central Node to Data Collection Module (Data Connection)

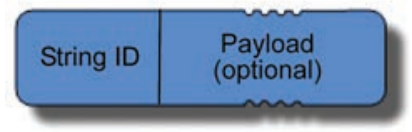

B) From Data Collection Module to Central Node (Control Connection)

Figure 7. Packet format used on the general packet radio service channel. 


\section{Power saving management}

To improve battery life, power consumption has been limited trying to achieve maximum efficiency while retaining all the features of the devices. For this purpose, a dedicated operating mode has been implemented: the devices are programmed to remain in a power down state until the execution of their specific functionalities requires them to be activated. In power down state the sensors and the transmitter are switched off, while the microcontroller is put in sleep mode, thus only preserving data in the internal memory and providing timer functionalities. Also, the power saving features of the ZED are fully exploited: the ZC caches packets to be sent to the $E D$ and sends them only upon an explicit request by the ZED. This request is performed on a regular basis by the $E D$. In the time between two consecutive connections (called ZigBee polling period) the ED switches his radio off while the microcontroller is in sleep mode.

Using this connection strategy allows considerable energy savings if compared to the case where the end device is still waiting for a polling from ZC. ZigBee polling period has been set to $1 \mathrm{~s}$ (though this parameter can be freely configured) to ensure an effective compromise between power saving and a prompt response of the devices in receiving data.

Energy consumption of the nodes, and lifetime of the batteries were estimated by means of laboratory tests. A system consisting of one $\mathrm{CN}$ and one SN equipped with a temperature sensor and a relative humidity sensor was used for the test; the SN was powered by three $1.5 \mathrm{~V} \mathrm{AA}$ type batteries with a capacity of $1100 \mathrm{mAh}$ each.

The test was carried out by evaluating the consumption of the SN device during every possible operational mode: i) sleep - a state characterised by very low consumption; ii) poll - network check and receipt of packets from the $\mathrm{CN}$; iii) wake-up - microcontroller wake-up; iv) battery measure - measurement of the battery voltage; v) sensor measure - reading of the sensors values; vi) process measure - elaboration of the measured values; vii) Tx data - data packet transmission to the $\mathrm{CN}$.

Time measurements carried out by means an oscilloscope were used to define the elapsed time of the different operational states.

\section{The test at the experimental greenhouse}

The field test was carried out, from March to September 2011, at the experimental centre of the University of Bari in Valenzano (Bari, Italy), latitude $41^{\circ} 05^{\prime} \mathrm{N}$. Climatic data were measured inside and outside a vaulted roof steel greenhouse $(10.00 \times 30.00 \mathrm{~m}$; ridge height of $4.45 \mathrm{~m}$; gutter height of $2.45 \mathrm{~m})$, North-South oriented.

The wireless monitoring system consisted of CN, RN, 4 SN's, and DCM (Figure 8). Two SN's were positioned inside the greenhouse, one of them (SN1) in central position, the other one (SN2) in the Southern part of the greenhouse; both the SN's were equipped with the Sensirion SHT75 sensor for air temperature and relative humidity measurement. The third SN (SN3) was positioned inside the greenhouse near the plants in order to measure the fruit diameter. The SN4 (Figure 9) was positioned outside the greenhouse and was equipped with the Schenk GmbH Model 8104 pyranometer for solar radiation measurement, the Sensirion SHT75 sensor for air temperature and relative humidity measurement, the Young Wind Sentry 03002 sensor for wind velocity and direction measurement, the Freescale MPX4115A sensor for absolute air pressure measurement.

The RN was positioned inside the greenhouse in the Southern part at about $14 \mathrm{~m}$ from the farthest $\mathrm{SN}$ inside the greenhouse and at about $11 \mathrm{~m}$ from the $\mathrm{CN}$, which was positioned inside a metallic box, outside the greenhouse.

Data were measured and collected by the wireless system at $120 \mathrm{~s}$ intervals.

Climatic data were also acquired by means of a system consisting of a data logger connected to the sensors by means of wires. The system measured solar radiation in the wavelength range $300-3000 \mathrm{~nm}$ by means of a pyranometer (model 8-48, Eppley Laboratory, Newport, RI, USA) with a uncertainty in instant measurement of $15 \mathrm{~W} \mathrm{~m}^{-2}$; wind velocity and direction by means of the Young Wind Sentry 03002 sensor; air temperature and relative humidity inside and outside the greenhouse by means of electronic sensors with an accuracy of $0.3^{\circ} \mathrm{C}$ and $1.5 \%$ respectively (Hygroclip-S3, Rotronic, Zurich, Switzerland). The data, measured at $60 \mathrm{~s}$ intervals, were averaged every $15 \mathrm{~min}$ and stored in the data logger (CR 10X, Campbell Scientific, Inc., Logan, UT, USA).

\section{Reliability of the radio-communication network}

The reliability of the radio-communication network was assessed by evaluating the data packets that were lost or un-correctly sent to the DCM server over the Internet. Data sent by two SN's (named 0x0301

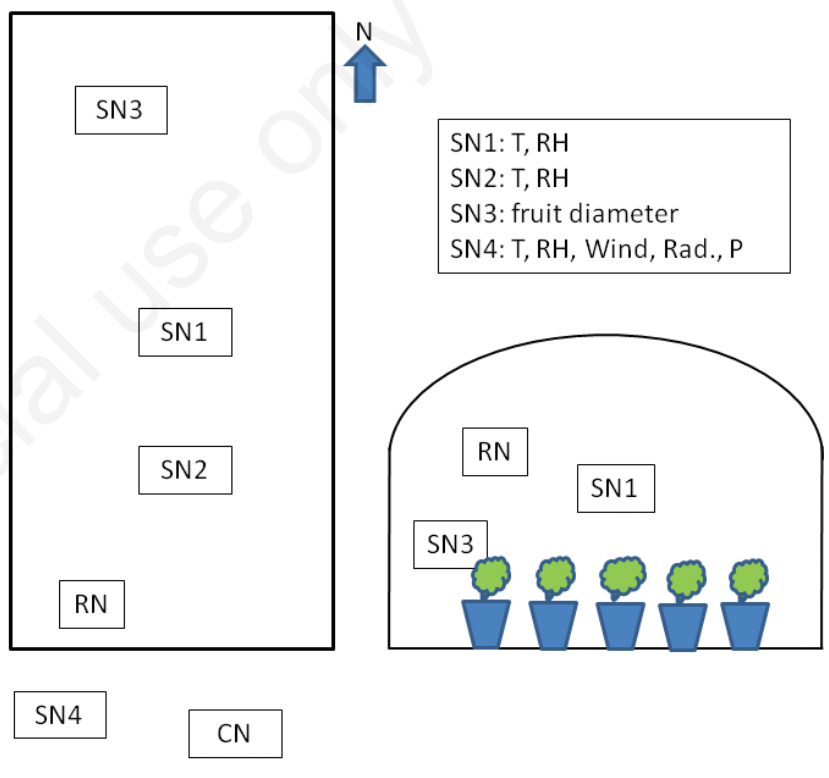

Figure 8. Layout of the nodes inside the greenhouse.

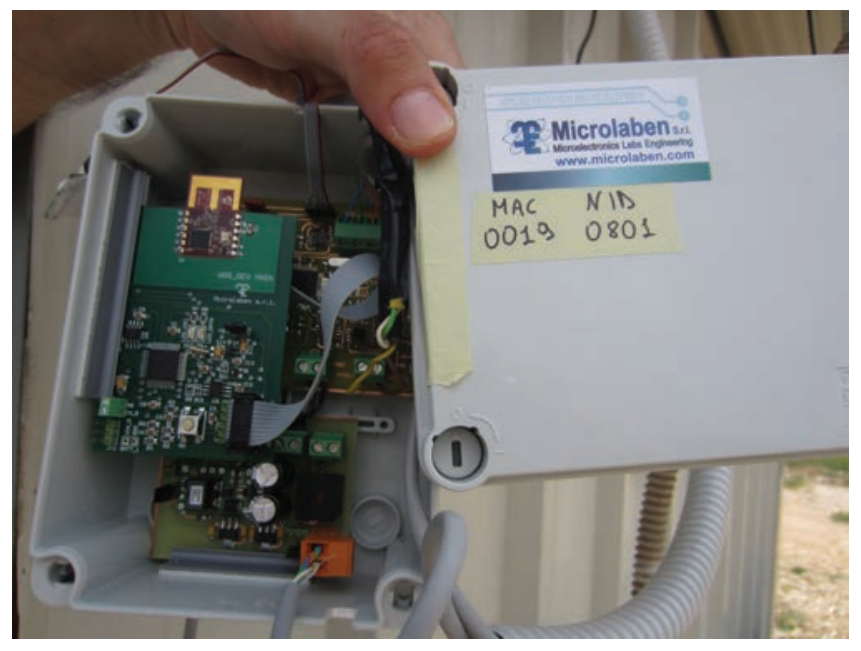

Figure 9. Sensor node. 
and $0 \times 0302$ ) located inside the greenhouse, equipped both with a temperature sensor and a relative humidity sensor, were analysed; the Pkt Period was equal to 120 s, i.e. 720 packets per day were exchanged. Data exchange was evaluated over an overall observation period of 20 days.

\section{Results}

Several performance indices have been investigated to assess the effectiveness of our system. First of all, an estimation of the battery life has been carried out for the SN's, considering the most useful configurations. Then, an estimate of the global system performance was car- ried out by analysing the reliability of the radio-communication network and the measurements accuracy.

\section{Power consumption evaluation}

Energy consumption of the nodes was estimated in the laboratory by using a system consisting of one CN and one SN equipped with a temperature sensor and a relative humidity sensor. Table 1 shows the current and power absorbed by the device and the measured elapsed time in the different states, the power consumption was calculated considering an average battery voltage equal to $4 \mathrm{~V}$. The elapsed time of the sleep period depends both on the network polling period (poll period) and on the data packet transmission period (Pkt period). Poll and Pkt period, which can be set during the working activities, determine the lifetime of the device battery.

Table 1. Current and power absorbed by the sensor node and elapsed time in the different operational states.

\begin{tabular}{lccc} 
State & Absorbed current (mA) & Absorbed power (mW) & Elapsed time (ms) \\
Sleep & 0.015 & 0.06 & 25.0 \\
Poll & 23.10 & 92.40 & 4.3 \\
\hline Wake-up & 4.10 & 16.40 & 21.0 \\
Battery measure & 4.10 & 16.40 & 265.0 \\
\hline Sensor measure & 0.56 & 2.240 & 0.8 \\
Process measure & 4.10 & 16.40 & 3.2 \\
\hline Tx Data & 27.10 & 108.40 & \\
\hline
\end{tabular}
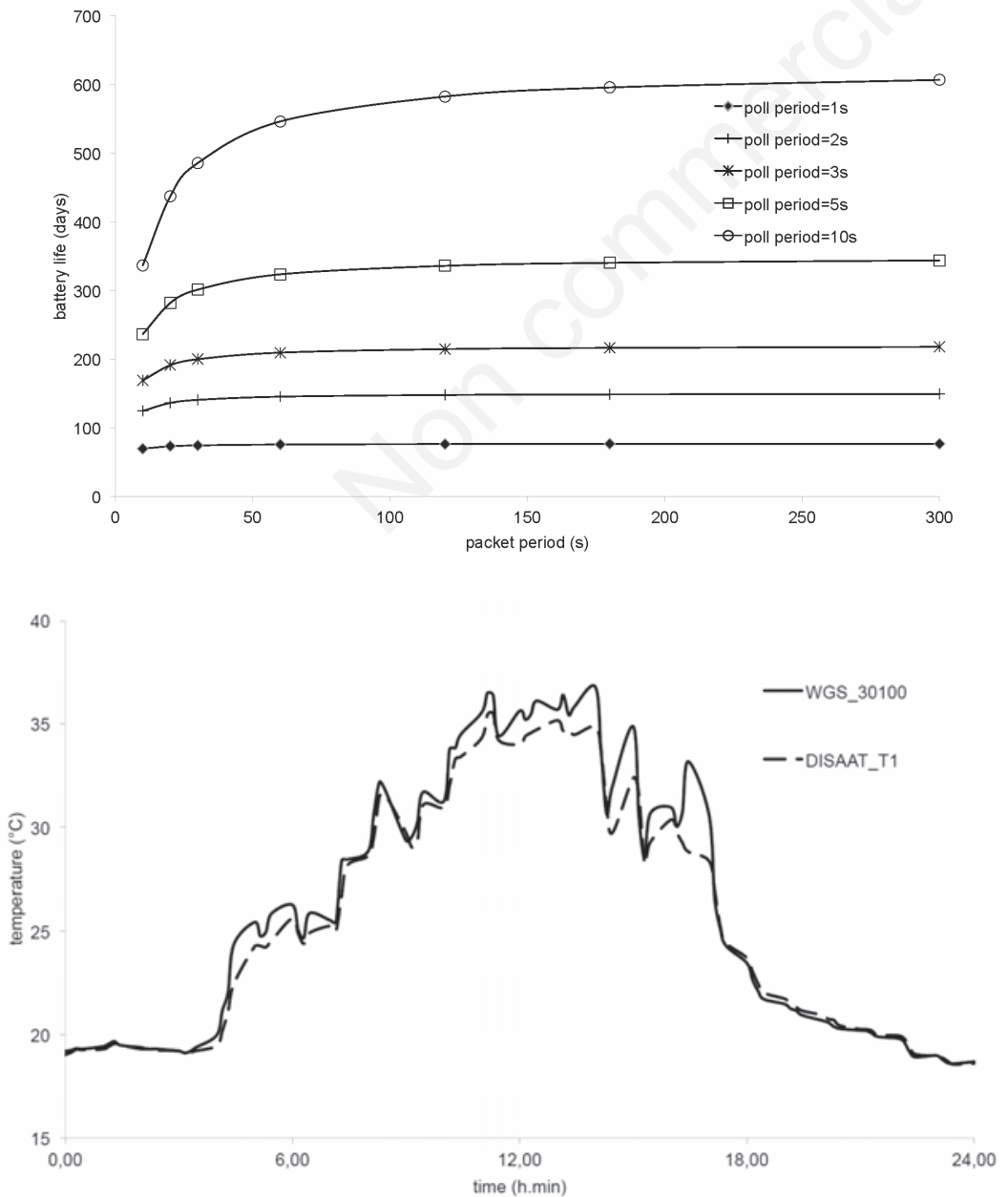

Figure 10. Battery lifetime of the node as a function of the packet and poll period.
Figure 11. Air temperature inside the greenhouse (central position) measured by the $\mathrm{SN} 1$ in the wireless system (WGS_30100) and by means of the wired system (DISAAT_T1). Measures carried out on $11 / 4 / 2011$. 
Based on the absorbed energy and on the working period of the states daily energy consumption and battery lifetime (Figure 10) were calculated as a function both of the poll period and of the Pkt period. Battery lifetime is influenced more by the poll period while the Pkt period mainly affects it in presence of the higher values of the poll period. The sensor node has a battery lifetime higher than 60 days in presence of the highest energy consumption that occurs with a Pkt period equal to $10 \mathrm{~s}$ and a poll period of $1 \mathrm{~s}$ (Figure 10). A Pkt period equal to $60 \mathrm{~s}$ and a poll period of 5 or $10 \mathrm{~s}$ are suitable values for greenhouse monitoring and control purposes, it means that the battery lifetime could range from 300 to 530 days.

\section{Reliability of the radio-communication network}

The test on the reliability of the radio-communication network was carried out, over an overall observation period of 20 days, by evaluating the data packets that were lost or un-correctly sent to the DCM server over the Internet .

The percentage of lost or un-correctly sent packets was equal to $1.5 \%$ for the $0 \mathrm{x} 0301$ node and equal to $1.6 \%$ for the $0 \mathrm{x} 0302$ node, over the observation period of the first 10 days. Based on the data collected during the first days some corrections were made to the system; some packets were lost at the same time by the two sensor nodes, it was related to a bug in the software that manages the communications over the Internet between the $\mathrm{CN}$ and the DCM and that caused the reset of the $\mathrm{CN}$. The software was corrected allowing the improvement of the communication; the percentage of lost or un-correctly sent packets decreased from $1.5 \%$ (first 10 days) to $0.3 \%$ for the $0 \mathrm{x} 0301$ node and from $1.6 \%$ (first 10 days) to $0.3 \%$ for the $0 \mathrm{x} 0302$ node, over the observation period of the second 10 days.

\section{Measurements accuracy}

Measurements made by the wireless system were collected by means of the DCM; the data were compared with the data acquired by means of the system consisting of sensors connected to the data logger by wires, used as control. The systematic error of the sensors was corrected by means of laboratory measurements carried out at the beginning and at the end of the field test, keeping the sensors in the same environmental conditions.

Data collected by means of the wireless system were collected and stored at $120 \mathrm{~s}$ intervals while the wired CR 10X Campbell data logger collected measurements at $60 \mathrm{~s}$ and stored them as average value every $900 \mathrm{~s}$. Figure 11 shows the comparison of the data, collected by the 2 systems, of the air temperature measured during one day inside the greenhouse in central position. Measurements realised by means of the wireless system are named WGS_30100, measurements carried out by means of the wired system are named DISAAT_T1. Data measured by the two systems were very similar, the mean value of the absolute difference of the two measures was equal to $0.60^{\circ} \mathrm{C}$, over an observation period of 10 days.

Figure 12 shows one day of measurements of air relative humidity carried out inside the greenhouse in central position; measurements realised by means of the wireless system are named WGS_30101,
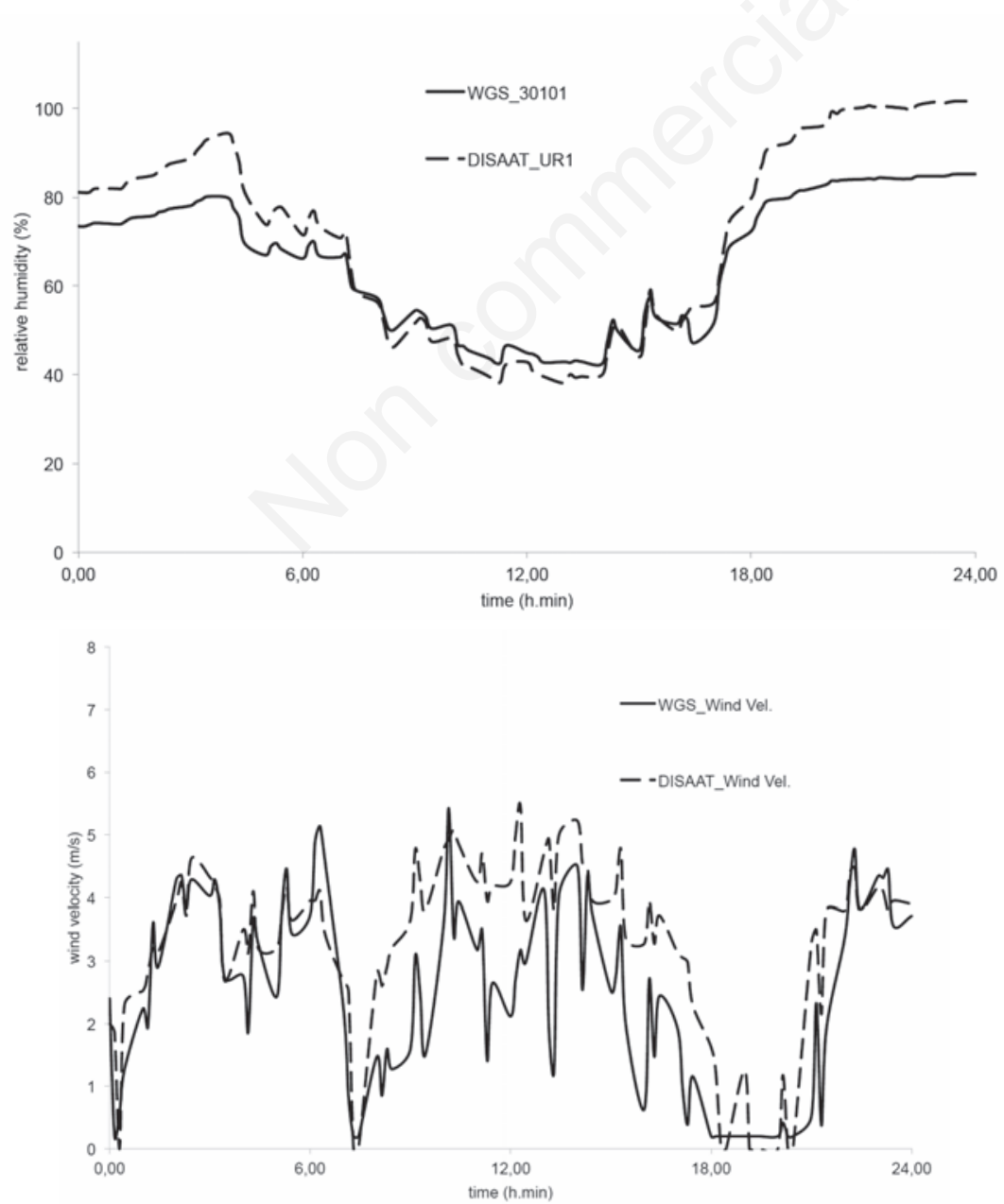

Figure 12. Air relative humidity inside the greenhouse measured by the SN1 in the wireless system (WGS 30101) and by means of the wired system (DISAAT UR1). Measures carried out on $11 / 4 / 2011$.
Figure 13. Wind velocity measured by the SN4 in the wireless system (WGS_Wind Vel.) and by means of the wired system (DISAAT_Wind Vel.). Measures carried out on 02/09/2011. 


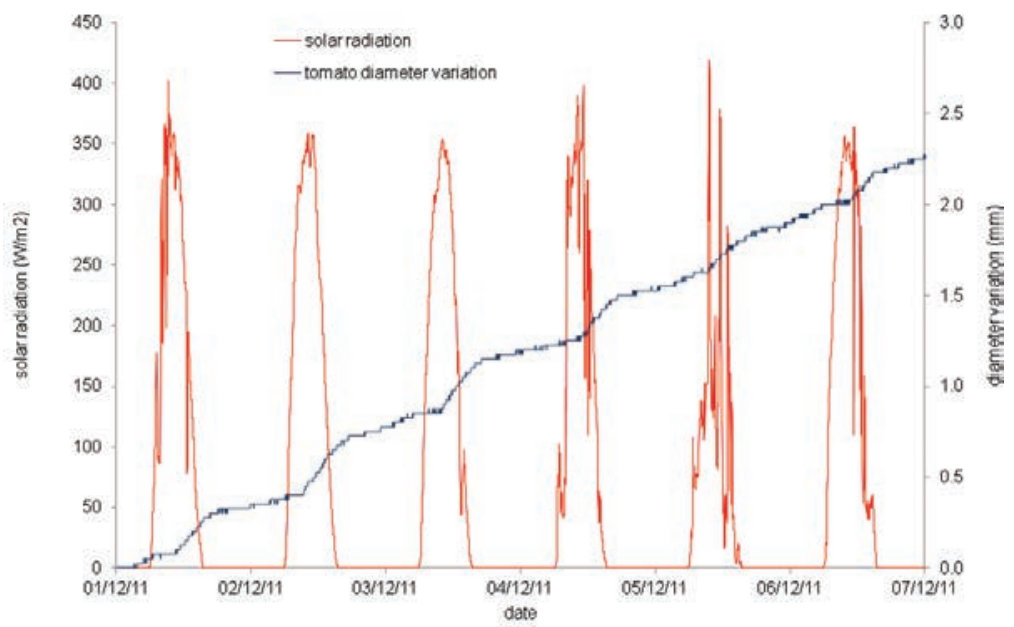

Figure 14. Variation of the tomato diameter and solar radiation.

measurements carried out by means of the wired system are named DISAAT_UR1; the data are similar during the day, higher differences were pointed out during the night. It is due to the different behaviour of the two sensors at high values of relative humidity. The mean value of the absolute difference of the two measures was equal to $7.77 \%$, calculated over an observation period of 10 days.

Figure 13 shows the comparison of the measurements of the wind velocity carried out by means of the wired (DISAAT_Wind Vel) and the wireless system (WGS_Wind Vel); the two systems used the same model of sensor, the mean value of the absolute difference of the two measures was equal to $0.78 \mathrm{~m} \mathrm{~s}^{-1}$, evaluated over an observation period of 10 days. Solar radiation was measured by the wireless and wired system using two different solar radiation sensors, the mean value of the absolute difference of the two measures, calculated over an observation period of 10 days, was equal to $6.28 \mathrm{Wm}^{-2}$.

\section{Fruit diameter sensor}

The fruit diameter sensor, integrated into the WSN, was used to measure the variation of the diameter of a tomato grown inside the greenhouse. Figure 14 shows the variation of the tomato diameter during six days in December together with the values of the solar radiation; the measured variation of the tomato diameter was $2.27 \mathrm{~mm}$; in the same period the maximum value of the solar radiation was about $400 \mathrm{Wm}^{-2}$ (Figure 14). The average values of the maximum and minimum daily greenhouse air temperature, calculated over the same six days, were $34.6^{\circ} \mathrm{C}$ and $8.7^{\circ} \mathrm{C}$, respectively, while the mean greenhouse air temperature was equal to $17.2^{\circ} \mathrm{C}$.

\section{Discussion}

The design of the system faced the main critical aspects that generally affect wireless systems. The tests carried out within the research showed that power consumption in sleep mode $(0.060 \mathrm{~mW})$ of the sensor node was lower than the value $(0.7125 \mathrm{~mW})$ recorded by the wireless system described by Garcia-Sanchez et al. (2011). Such low values of energy consumption allowed a lifetime of the sensor node battery higher than 500 days.

Concerning the reliability of the communication network the system showed a percentage of lost or un-correctly sent packets equal to $0.3 \%$; Garcia-Sanchez et al. (2011) found that about $2 \%$ of the sent message were lost during the communication between the system in the field and the server that performed data collection.

Data measured and collected by the wireless system were compared with the data measured with the wired system; the mean value of the difference in air temperature measurement, equal to $0.60^{\circ} \mathrm{C}$, can be attributed to the use of two different temperature sensors, to the different sampling period and to the air temperature gradients that characterise the greenhouse microclimate (Teitel et al., 2010). The measurements of the wind velocity by means of the two systems, carried out by using the same model of sensor, showed a mean difference equal to $0.78 \mathrm{~m} \mathrm{~s}^{-1}$, which can be attributed to the different sampling time and that was anyway lower than the sensor accuracy, equal to $1.1 \mathrm{~m} \mathrm{~s}^{-1}$ (R.M. Young Company, 1999).

The use of an innovative fruit diameter sensor within the Wireless Sensor Network was designed in order to make the monitoring system applicable in innovative greenhouse control and management strategies such as the speaking plant approach (Morimoto and Hashimoto, 2009).

\section{Conclusions}

High added-value greenhouse agricultural production requires the achievement of optimal greenhouse microclimate conditions that relies on a reliable monitoring system. A greenhouse microclimate monitoring system must have peculiar features related to its flexibility and reliability; the units composing the system, indeed, must be located in different parts of the greenhouse. Wireless network and battery-powered components can be used to overcome the problems related to wires cabling, i.e. presence of a dense net of wires crossing the cultivation area and hampering the cultivation practices, wires subjected to high temperature and relative humidity together with thermal cycling, and animals such as voles that can damage the wires.

The paper presents a wireless system designed for monitoring greenhouse ambient parameters and the experimental results of its application in real conditions. During the test in the field the wireless monitoring system showed a good performance in terms of energy consumption, reliability of the radio communication network and accuracy of the measurements. The system has proven to be flexible enough to guarantee the best trade-off between responsiveness and power consumption; the tests showed that a battery lifetime of 530 days can be obtained for a sensor node. Research output was the definition of the working parameters specific for greenhouse applications, i.e. the network polling period and the data packet transmission period.

The use of wireless technologies makes possible to guarantee reliable operation and ease of deployment, thanks to the IEEE 802.15.4 and GPRS wireless technologies. Sensors for the measurement of climatic parameters such as solar radiation, air temperature and relative 
humidity were integrated in the system, besides the wireless system allows the integration of innovative sensors such as fruit diameter sensors, which can be deployed on the plants without wires. Such systems can be applied in intelligent cultivation control techniques to regulate, for example, the irrigation system in relation with the fruit diameter.

Future development of the research should be addressed to develop wireless climate measurement and control systems, including both sensors and equipment actuators.

\section{References}

Analog Devices Inc. 2014. ADIS16201 Programmable dual-axis inclinometer/accelerometer -Data sheet. Available from: http://www.analog.com/static/imported-files/data_sheets/ADIS 16201.pdf

Baronti P., Pillai P., Chook V.W., Chessa S., Gotta A., Fu Y.F. 2007. Wireless sensor networks: a survey on the state of the art and the 802.15.4 and ZigBee standards. Comput. Comm. 30:1655-95.

Bartzanas T., Tchamitchian M., Kittas C. 2005. Influence of the heating method on greenhouse microclimate and energy consumption. Biosyst. Eng. 91:487-99.

Bot G.P.A. 2001. Developments in indoor sustainable plant production with emphasis on energy saving. Comput. Electron. Agric. 30:151-65.

Freescale Semiconductor. 2014. MPXx4115: -115 to $115 \mathrm{kPa}$ vacuum integrated pressure sensor. Available from: http://www.freescale. com/webapp/sps/site/prod_summary.jsp? code=MPXx4115\&fsrch $=1$ $\& s r=1 \&$ pageNum $=1$

Garcia-Sanchez A.J., Garcia-Sanchez F., Garcia-Haro J. 2011. Wireless sensor network deployment for integrating video-surveillance and data-monitoring in precision agriculture over distributed crops. Comput. Electron. Agric. 75:288-303.

IEEE Standard Association. 2006. IEEE 802.15.4. Wireless Medium Access Control (MAC) and Physical Layer (PHY) Specifications for Low-Rate Wireless Personal Area Networks (WPANs). The Institute of Electrical and Electronics Engineers Inc., New York, NY, USA.

Li X.-H., Cheng X., Yan K., Gong P. 2010. A monitoring system for vegetable greenhouses based on a wireless sensor network. Sensors 10:8963-80.

López Riquelme J.A., Soto F., Suardíaz J., Sánchez P., Iborra A., Vera J.A. 2009. Wireless sensor networks for precision horticulture in Southern Spain. Comput. Electron. Agric. 68:25-35.

Matese A., Di Gennaro S.F., Zaldei A., Genesio L., Vaccari F.P. 2009. A wireless sensor network for precision viticulture: The NAV system. Comput. Electron. Agric. 69:51-8.

Microchip Technology Inc. 2014. PIC24H microcontroller. Available from: http://www.microchip.com

Morandi B., Manfrini L., Zibordi M., Moferini M., Fiori G., Corelli Grappadelli L. 2007. A low-cost device for accurate and continous measurements of fruit diameter. Hort. Sci. 42:1380-2.

Morimoto T., Hashimoto Y. 2009. Speaking plant/fruit approach for greenhouse and plant factories. Environ. Control Biol. 47:55-72.

Nadimi E.S., Søgaard H.T., Bak T. 2008. ZigBee-based wireless sensor networks for classifying the behaviour of a herd of animals using classification trees. Biosyst. Eng. 100:167-76.

Novello V., De Palma L., Tarricone L., Vox G. 2000. Effects of different plastic sheet coverings on microclimate and berry ripening of table grape CV 'Matilde'. J. Int. Sci. Vigne Vin. 34:49-55.

Papadakis G., Briassoulis D., Scarascia Mugnozza G., Vox G., Feuilloley P., Stoffers J.A. 2000. Radiometric and thermal properties of, and testing methods for, greenhouse covering materials. J. Agr. Eng. Res. 77:7-38.

Picuno P., Tortora A., Capobianco R.L. 2011. Analysis of plasticulture landscapes in Southern Italy through remote sensing and solid modeling techniques. Landscape Urban Plan. 100:45-56.

R.M. Young Company. 1999. Wind Sentry 03002 Data sheet. Avaialble from: http://www.youngusa.com/

Ruiz-Garcia L., Barreiro P., Robla J.I. 2008. Performance of ZigBeeBased wireless sensor nodes for real-time monitoring of fruit logistics. J. Food Eng. 87:405-15.

Schettini E., De Salvador F.R., Scarascia Mugnozza G., Vox G. 2011. Radiometric properties of photoselective and photoluminescent greenhouse plastic films and their effects on peach and cherry tree growth. J. Horticult. Sci. Biotechnol. 86:79-83.

Schettini E., Vox G. 2012. Effects of agrochemicals on the radiometric properties of different anti-UV stabilized EVA plastic films. Acta Hortic. 956:515-22.

Sensirion AG. 2014. Data Sheet SHT7x (SHT71, SHT75) - Humidity and temperature sensor IC. Available from: http://www.sensirion. com/nc/en/products/humidity-temperature/ download-center/?cid= $8574 \&$ did $=68 \&$ sechash $=2$ f9d $5 \mathrm{~b} 5 \mathrm{c}$

Sica C., Picuno P. 2008. Spectro-radiometrical characterization of plastic nets for protected cultivation. Acta Hortic. 801:245-52.

Teitel M., Atias M., Barak M. 2010. Gradients of temperature, humidity and C02 along a fan-ventilated greenhouse. Biosyst. Eng. 106:166-74.

Telit. 2014. GM862 product description. Available from: http://www. telit.co.it

The Python Programming Language. 2014. Python packaging user guide. Available from: http://www.python.org

The 3rd Generation Partnership Project (3GPP). 2014. Std. General Packet Radio Service (GPRS). Available from: http://www.3gpp.org

Valente F., Zacheo G., Losito P., Camarda P. 2009a. A telecommunications framework for real-time monitoring of dangerous goods transport. Page 6 in Proc. ITS-T 2009, Lille, France.

Valente F., Zacheo G., Losito P., Corsi F. 2009b. A two-tier hierarchical network for adverse event monitoring. Page 6 in Proc. IWASI 2009, Trani, Italy.

Vox G., Schettini E., Scarascia-Mugnozza G. 2005. Radiometric properties of biodegradable films for horticultural protected cultivation. Acta Hortic. 691:575-82.

Vox G., Schettini E., Lisi Cervone A., Anifantis A. 2008. Solar thermal collectors for greenhouse heating. Acta Hortic. 801:787-94.

Vox G., Teitel M., Pardossi A., Minuto A., Tinivella F., Schettini E. 2010. Chapter 1: Sustainable greenhouse systems. In: A. Salazar and I. Rios (Eds.), Sustainable agriculture: technology, planning and management. Nova Science Publishers, Inc., New York, NY, USA. Available from: https://www.novapublishers.com/catalog/product_ info.php?products_id=17788

Vox G., Scarascia Mugnozza G., Schettini E., de Palma L., Tarricone L., Gentilesco G., Vitali M. 2012. Radiometric properties of plastic films for vineyard covering and their influence on vine physiology and production. Acta Hortic. 956:465-72.

Zhou Y., Yang X., Guo X., Zhou M., Wang L. 2007. A design of greenhouse monitoring \& control system based on ZigBee wireless sensor network. pp 2563-2567 in Proc. Int. Conf. on Wireless Communications, Networking and Mobile Computing, WiCom 2007, 21-25 Sept. 2007, Shanghai, China

ZigBee Alliance. 2014. Specifications. Available from: http://www.zigbee.org/Specifications.aspx 